\title{
Free vibration analysis of a string with vibration suppressor (When the position of vibration suppressor is opposite to the pulled position)
}

\author{
Hiroyuki KIMURA* \\ *Graduate School of Science and Engineering for Research (Engineering), Toyama University \\ 3190 Gofuku, Toyama-shi, Toyama 930-8555, Japan \\ E-mail: hkimura@eng.u-toyama.ac.jp
}

Received 8 October 2015

\begin{abstract}
A vibration suppressor is used to change the natural frequency of an elevator rope, and to prevent resonance. The elevator rope is modeled using a string. However, the vibration of the string that has a vibration suppressor has been studied for a few conditions because of its geometric nonlinearity. In this paper, an exact solution for the free vibration of a string when the position of the vibration suppressor is opposite to the pulled position is presented. In this analysis, a problem of free vibration with a vibration suppressor is transposed to a problem of forced vibration. Further, to verify the validity of this exact solution, a finite difference analysis of the string vibration with a vibration suppressor is performed. The calculated results obtained from the finite difference analysis are in good agreement with those of the exact solution.
\end{abstract}

Key words : String, Free vibration, Vibration suppressor, Exact solution, Elevator rope, Rope sway

\section{Introduction}

When an elevator rope of a high-rise building is forcibly excited by the displacements of the building induced by wind forces and/or by long-period ground motion, the rope displacement becomes large, even if the ground and the building accelerations are small. To prevent this problem, the elevator should be driven within a certain limit depending on the sway of the building. Thus, to improve the driving efficiency and safety of the elevator, the rope sway should be reduced.

As shown in Fig. 1, the elevator rope is composed of a main rope and a compensating rope, where the cage and counterweight are hung from the main rope. A compensating sheave and a compensating rope ensure the tension on the main rope. Studies that seek to reduce the lateral vibration of an elevator rope have been reported in the literature; for example, a control method involving the consideration of the time-varying rope length (Otsuki et al., 2002); numerical analysis of the elevator rope using a vibration suppressor (Kimura et al., 2002) (Kimura and Nakagawa, 2007a); reducing method involving the movement of the cage location to avoid resonance caused by the sway of building (Watanabe et. al., 2007); non-contact control method using a permanent magnet (Katsuno et al., 2007); and another reducing method using a damper attached to the compensating sheave (Nakagawa and Arai, 2012). Only vibration suppressors that physically restrain rope displacement are used in elevator systems to reduce rope vibration (Nakagawa et al., 2005).

The vibration of a string that has a vibration suppressor has been studied only for a few conditions because of its geometric nonlinearity (Fig. 2). The author has analyzed the vibration of an elevator rope that has a vibration suppressor, assuming that the rope is a string. The advantage of using a vibration suppressor for reducing the lateral vibration of the rope is demonstrated through numerical calculations. The effects of the vibration suppressor greatly depend on its position, number and gap between the string and suppressor (Kimura et al., 2002) (Kimura and Nakagawa, 2007a). If a theoretical solution is obtained for rope sway due to forced vibration, the optimal installation position and the number of vibration suppressors can be determined without conducting a numerical calculation. 
Next, the free vibration of the string with vibration suppressors as shown in Fig. 2 has been studied and exact solutions have been obtained. For the case where the vibration suppressors are uniformly installed and the pulled position is at the center of the string (Fig. 2(a)), the effects of the gap and number of vibration suppressors are presented (Kimura, 2013). The effect of the number of vibration suppressors is presented for the case when the vibration suppressors are uniformly installed and the pulled position is $1 / N$ of the position of the string (Fig. 2(b)) (Kimura, 2014a) where $N$ is divided number of the string. Further, the exact solution for the free vibration of the string with one vibration suppressor is presented for the case when the $1 / N$ position of the string is pulled (Fig. 2(c), (d)) (Kimura, 2014a, 2014b). However, in the case where the position of the vibration suppressor is opposite to the pulled position, no exact solution has yet been obtained for the free vibration of the string.

This study determines the exact solution for the free vibration of a string when the position of the vibration suppressor is opposite to the pulled position. In addition, a finite difference analysis of the string vibration is performed to verify the validity of the exact solution.

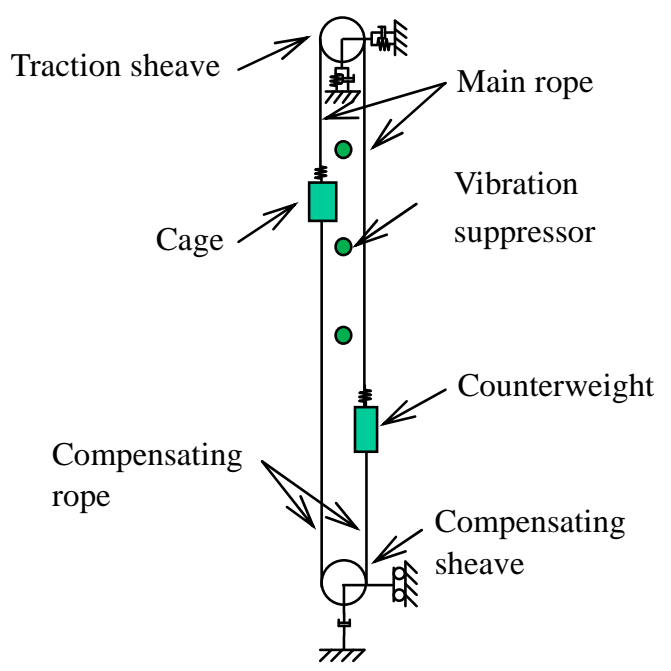

Fig. 1 Schematic diagram of elevator rope system

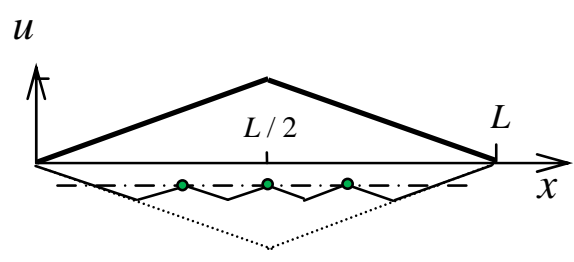

(a) $L / 2, N=4, N_{s}=N-1$

$u$

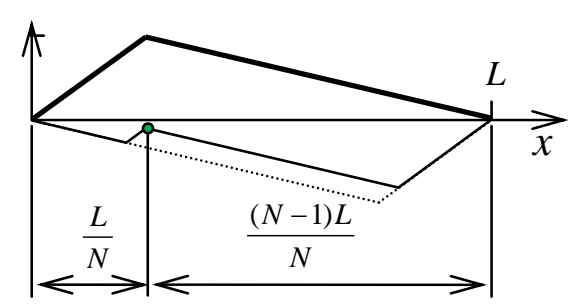

(c) $L / N, N=4, N_{s}=1$

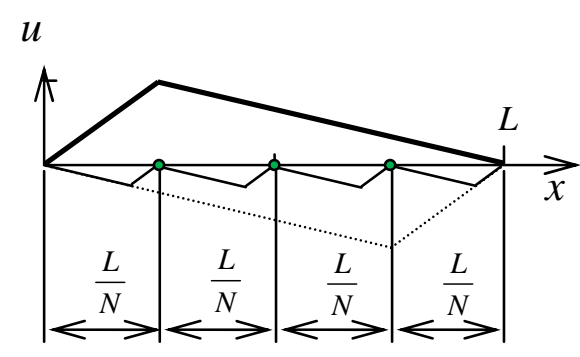

(b) $L / N, N=4, N_{s}=N-1$ $u$

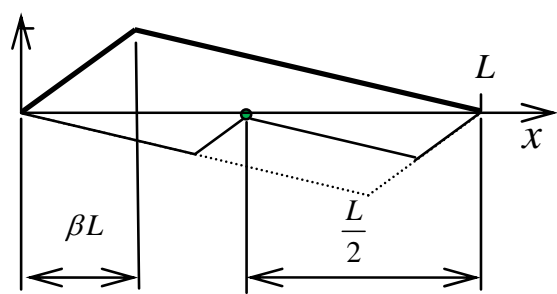

(d) $\beta L, \beta=0.25, N_{s}=1$

Fig. 2 Initial string displacement and vibration suppressors (pulled position, divided number, number of vibration suppressors). $L$ and $u$ are the string length and string displacement ,respectively. $L / N$ and $\beta L$ are the pulled position. 


\section{Analytical Method}

\subsection{Exact Solution}

This study determines the free vibration of a string that is released after an initial displacement, as shown in Fig. 3. A vibration suppressor is in an opposite position to the pulled position. Assuming that the string tension is constant and the initial velocity of the string is zero, the initial displacement is expressed as follows:

$$
u(x, 0)= \begin{cases}\frac{1}{\beta} \cdot \frac{x}{L} u_{\max } & (0 \leq x \leq \beta L) \\ \frac{1}{1-\beta}\left(1-\frac{x}{L}\right) u_{\max } & (\beta L \leq x \leq L)\end{cases}
$$

where $L$ is the string length, $u_{\max }$ is the initial displacement at the pulled position, and $\beta L$ is the pulled position.

The boundary condition is expressed as follows:

$$
u \geq-\delta_{0} \quad(\text { at } \quad x=(1-\beta) L)
$$

where $\delta_{0}$ is the gap between the string and the vibration suppressor.

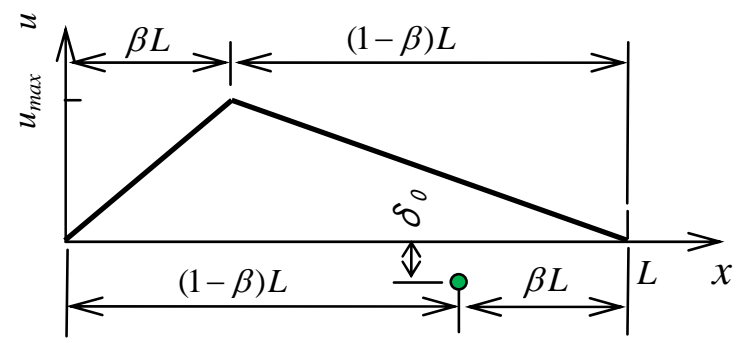

Fig. 3 Initial string displacement

\subsubsection{Zero gap between the string and the vibration suppressor}

The displacement of the string without the vibration suppressor is shown in Fig. 4(a). The initial displacement is plotted in blue. After releasing the string, the string displacement changes as shown by lines DAF, DEF, DGF, DHF, DGF, and DEF, and it returns to the initial position (line DAF). The displacement of the string with a vibration suppressor is shown in Fig. 4(b). The wave propagation in the string is shown in Fig. 5, where $\beta$ is equal to $1 / 3$, and $\delta_{0}$ is equal to zero. The situation is the same as that for the case where there is no vibration suppressor until the string collides with the vibration suppressor $\left(t<T_{A}\right)$. The string displacement at point $\mathrm{B}\left(t=T_{A}\right)$ is plotted in red. Time $T_{A}$ is expressed as in the following equation using a geometrical relation as shown in Fig. 4(b). Time $T_{A}$ is equal to $T_{A}^{\prime}$ which is the time taken by the string to return to the initial string displacement.

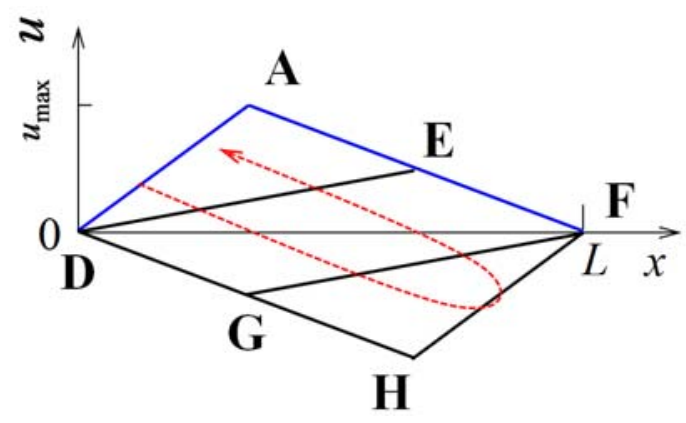

(a) Without vibration suppressor

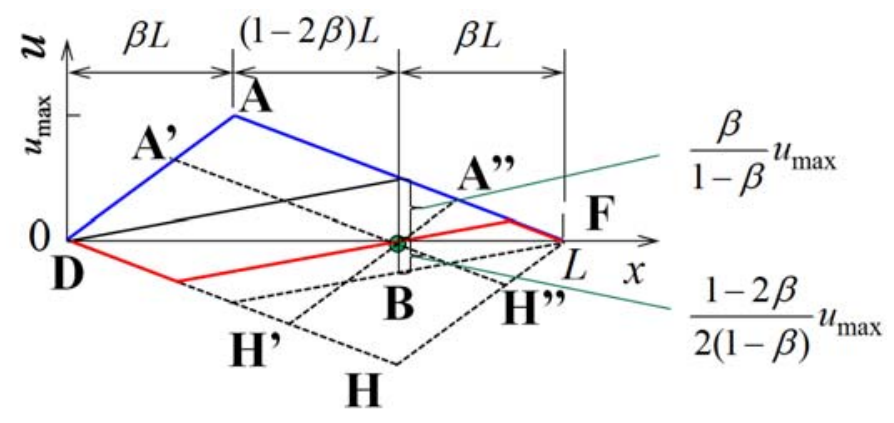

(b) With vibration suppressor

Fig. 4 Displacement of string with/without vibration suppressor 


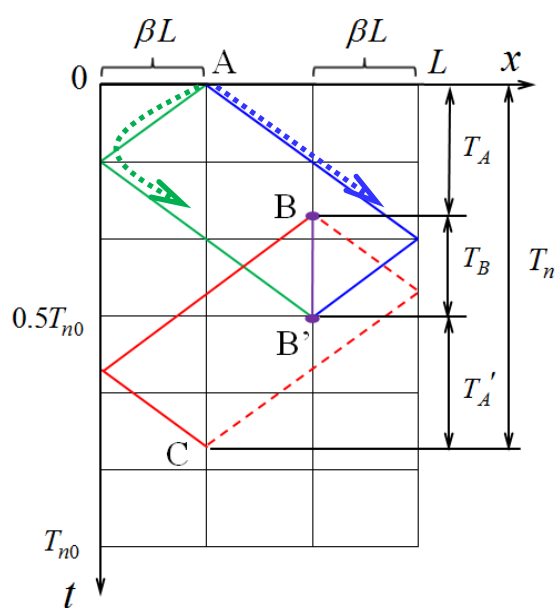

Fig. 5 Propagation waves of string $\left(\beta=1 / 3, \delta_{0}=0\right)$

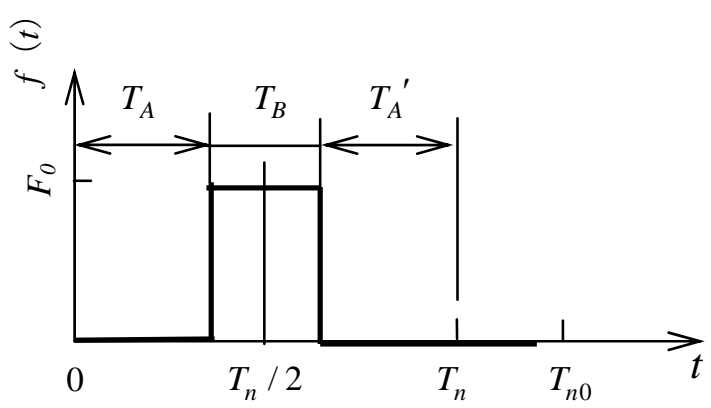

Fig. 6 Time history of reaction force

$$
\left(\beta=1 / 3, \quad \delta_{0}=0\right)
$$

$$
T_{A}=T_{A}^{\prime}=\frac{T_{n 0}}{2 L}\left\{(1-2 \beta) L+\beta L \frac{\frac{\beta}{1-\beta} u_{\max }}{\frac{\beta}{1-\beta} u_{\max }+\frac{1-2 \beta}{2(1-\beta)} u_{\max }}\right\}=\frac{\{1-2 \beta(1-\beta)\}}{2} T_{n 0}
$$

where $T_{n 0}$ is the natural period without the vibration suppressor.

Straight lines in Fig. 5 indicate the wave propagation in the string. The wave from point $\mathrm{A}(t=0)$ propagates to the right and left and reflects at both ends, while the string collides with the vibration suppressor at point $\mathrm{B}\left(t=T_{A}\right)$. Then, a new wave propagates from point B. The load acts on the vibration suppressor from the string between B and B' in Fig. 5. Figure 6 shows the time history of the load. The same load acts on the string from the vibration suppressor.

Time $T_{B}$ is expressed as in the following equation considering the wave propagation as shown in Fig. 5.

$T_{B}=0.5 T_{n 0}-T_{A}=\beta(1-\beta) T_{n 0}$

Parallelograms ADHF, A'DH'B, and A"BH"F are similar in Fig. 4. Angle H'BH" is equal to angle DAF, because the corresponding angles of similar parallelograms are equal. Further, angle $\mathrm{H}^{\prime} \mathrm{BH}^{\prime \prime}$ is constant from point B to B'. Therefore, the amplitude of the load is constant. String displacement at point B' is equal to the displacement (red line in Fig. 4(b)) at point B, and returns to the initial displacement.

Thus, the natural period $T_{n}$ and the natural frequency $f_{n}$ are expressed as follows:

$$
\begin{aligned}
& T_{n}=T_{A}+T_{B}+T_{A}^{\prime}=\{1-\beta(1-\beta)\} T_{n 0} \\
& f_{n}=\frac{1}{1-\beta(1-\beta)} f_{n 0}
\end{aligned}
$$

where $f_{n 0}$ is the natural frequency without the vibration suppressor.

As described above, string vibration with vibration suppressor is able to trace by considering the wave propagation in the string. However, in the case of this method, it's necessary to change the equation according to coordinate and time. In this study, it's expressed in one equation as shown below.

The magnitude of the load is expressed as $F_{0}=T_{0} \cdot u_{\max } /(L \cdot \beta(1-\beta))$ using the initial displacement $u_{\max }$ and the string tension $T_{0}$. In addition, there should be no energy loss due to collision.

The load $f(t)$ is expressed as follows:

$$
f(t)=\left\{\begin{array}{lc}
0 & \left(0<t<T_{A}\right) \\
F_{0} & \left(T_{A}<t<T_{A}+T_{B}\right) \\
0 & \left(T_{A}+T_{B}<t<T_{n}\right)
\end{array}\right.
$$

Because the load $f(t)$ is an even function (Fig.6), the Fourier series expansion of $f(t)$ is obtained as follows:

$$
f(t)=F_{0}\left\{\frac{\beta(1-\beta)}{1-\beta(1-\beta)}-\frac{2}{\pi} \sum_{k=1}^{\infty} \frac{1}{k} \cdot \sin \frac{1-2 \beta(1-\beta)}{1-\beta(1-\beta)} k \pi \cdot \cos \frac{2 k \pi t}{\{1-\beta(1-\beta)\} T_{n 0}}\right\}
$$


Therefore, the problem of this free vibration with a vibration suppressor can be transposed to the problem of forced vibration on the concentrated load, which is expressed in Eq. (9). This concentrated load acts at $x=(1-\beta) L$.

$$
f(x, t)=F_{0} \delta(x-(1-\beta) L)\left\{\frac{\beta(1-\beta)}{1-\beta(1-\beta)}-\frac{2}{\pi} \sum_{k=1}^{\infty} \frac{1}{k} \cdot \sin \frac{1-2 \beta(1-\beta)}{1-\beta(1-\beta)} k \pi \cdot \cos \frac{2 k \pi t}{\{1-\beta(1-\beta)\} T_{n 0}}\right\}
$$

where $\delta(x-(1-\beta) L)$ is the Dirac delta function. The constant term $F_{0} \delta(x-(1-\beta) L) \cdot \beta(1-\beta) /\{1-\beta(1-\beta)\}$ in Eq. (9) corresponds to the static load. The solution is expressed as follows:

$$
u_{1}(x, t)= \begin{cases}\frac{\beta}{1-\beta(1-\beta)} \times \frac{x}{L} u_{\max } & (0 \leq x \leq(1-\beta) L) \\ \frac{1-\beta}{1-\beta(1-\beta)} \times\left(1-\frac{x}{L}\right) u_{\max } & ((1-\beta) L \leq x \leq L)\end{cases}
$$

The solution corresponding to the remaining terms of Eq. (9) is obtained as the following equation by applying the solution to the forced vibration where a sinusoidal load acts at the center of the string (Yasuda, 2000).

$$
\begin{aligned}
u_{2}(x, t)= & -\frac{4 u_{\max }}{\beta(1-\beta) \pi^{3}} \sum_{k=1}^{\infty} \sum_{i=1}^{\infty}\left[\frac{1}{i^{2}-\left(\frac{k}{1-\beta(1-\beta)}\right)^{2}} \cdot \frac{1}{k} \cdot \sin \frac{1-2 \beta(1-\beta)}{1-\beta(1-\beta)} k \pi \cdot \sin (1-\beta) i \pi\right. \\
& \left.\times \sin \frac{i \pi x}{L} \cdot \cos \frac{2 k \pi t}{(1-\beta(1-\beta)) T_{n 0}}\right]
\end{aligned}
$$

Therefore, when the pulled position is opposite to the position of the vibration suppressor and the gap $\delta_{0}$ is zero, the exact solution for the free vibration of the string is expressed as follows:

$$
u(x, t)=u_{1}(x, t)+u_{2}(x, t)
$$

\subsubsection{Non-zero gap $\delta_{0}$}

Next, we considered the case where the gap $\delta_{0}$ is not limited to zero. Figure 7 shows the propagation waves of the string after the string is released. The difference between Fig. 7 and Fig. 5 is the collision time (position of point B). As shown in Fig. 8, the load acts on the vibration suppressor. Thus, as noted in the foregoing paragraph, the exact solution is obtained as follows:

$$
\begin{aligned}
& T_{A}=T_{A}{ }^{\prime}=\frac{T_{n 0}}{2 L}\left\{(1-2 \beta) L+\beta L \frac{\left(\frac{\beta}{1-\beta}+\alpha\right) u_{\max }}{\left(\frac{\beta}{1-\beta}+\alpha\right) u_{\max }+\left(\frac{1-2 \beta}{2(1-\beta)}-\alpha\right) u_{\max }}\right\}=\frac{\{1-2 \beta(1-\beta)(1-\alpha)\}}{2} T_{n 0} \\
& T_{B}=0.5 T_{n 0}-T_{A}=\beta(1-\beta)(1-\alpha) T_{n 0} \\
& T_{n}=T_{A}+T_{B}+T_{A}^{\prime}=\{1-\beta(1-\beta)(1-\alpha)\} T_{n 0} \\
& f_{n}=\frac{1}{\{1-\beta(1-\beta)(1-\alpha)\}} f_{n 0} \\
& f(x, t)=F_{0} \delta(x-(1-\beta) L) \\
& \quad \times\left\{\frac{\beta(1-\beta)(1-\alpha)}{1-\beta(1-\beta)(1-\alpha)}-\frac{2}{\pi} \sum_{k=1}^{\infty} \frac{1}{k} \cdot \sin \frac{1-2 \beta(1-\beta)(1-\alpha)}{1-\beta(1-\beta)(1-\alpha)} k \pi \cdot \cos \frac{2 k \pi t}{\{1-\beta(1-\beta)(1-\alpha)\} T_{n 0}}\right\}
\end{aligned}
$$




$$
\begin{aligned}
u_{1}(x, t)= & \begin{cases}\frac{\beta(1-\alpha)}{1-\beta(1-\beta)(1-\alpha)} \times \frac{x}{L} u_{\max } & (0 \leq x \leq(1-\beta) L) \\
\frac{(1-\beta)(1-\alpha)}{1-\beta(1-\beta)(1-\alpha)} \times\left(1-\frac{x}{L}\right) u_{\max } & ((1-\beta) L \leq x \leq L)\end{cases} \\
u_{2}(x, t)= & -\frac{4 u_{\max }}{\beta(1-\beta) \pi^{3}} \sum_{k=1}^{\infty} \sum_{i=1}^{\infty}\left[\frac{1}{i^{2}-\left(\frac{k}{1-\beta(1-\beta)(1-\alpha)}\right)^{2}} \cdot \frac{1}{k} \cdot \sin \frac{1-2 \beta(1-\beta)(1-\alpha)}{1-\beta(1-\beta)(1-\alpha)} k \pi\right. \\
& \left.\times \sin (1-\beta) i \pi \cdot \sin \frac{i \pi x}{L} \cdot \cos \frac{2 k \pi t}{\{1-\beta(1-\beta)(1-\alpha)\} T_{n 0}}\right]
\end{aligned}
$$

where $\alpha=\delta_{0} / u_{\max }$.

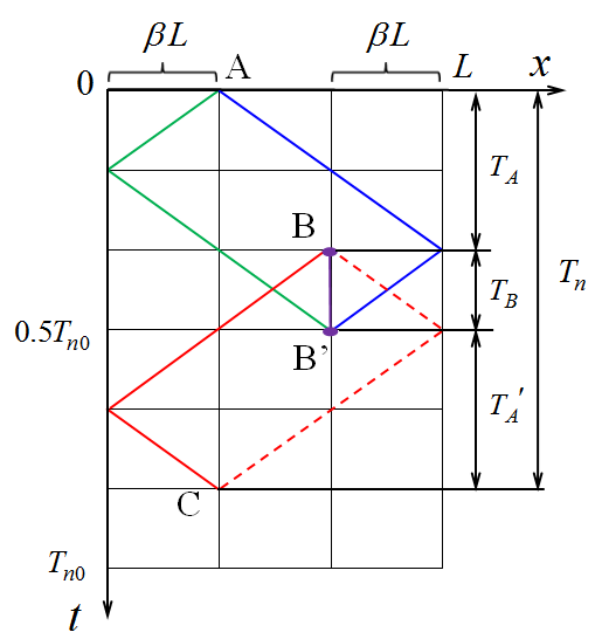

Fig. 7 Propagation waves of string ( $\beta=1 / 3, \quad \alpha=0.25$ )

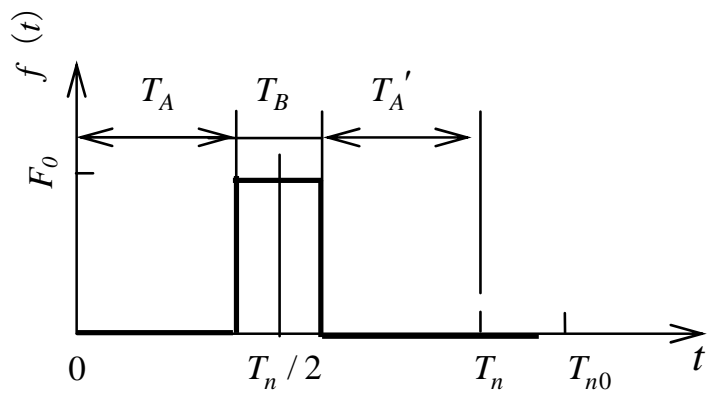

Fig. 8 Time history of reaction force

$$
(\beta=1 / 3, \quad \alpha=0.25)
$$

Therefore, when the pulled position is opposite to the position of the vibration suppressor and the gap $\delta_{0}$ is not limited to zero, the exact solution for the free vibration of the string is expressed as follows:

$$
u(x, t)=u_{1}(x, t)+u_{2}(x, t)
$$

\subsection{Equation of motion and finite difference analysis}

Assuming that string length and tension are constant, the differential equation of the string can be expressed as follows:

$$
\rho A \frac{\partial^{2} u}{\partial t^{2}}-T_{0} \frac{\partial^{2} u}{\partial x^{2}}+C \frac{\partial u}{\partial t}=0
$$

where $x$ is the coordinate of the string, $\rho A$ is the mass density per unit length, $C$ is the damping coefficient per unit length, and $T_{0}$ is the string tension.

This study investigates a string with one vibration suppressor. A numerical analysis is performed using the following difference equation (Kimura et al., 2007b, 2008), derived from Eq. (21). 


$$
\left(1+\frac{C}{\rho A} \frac{\Delta t}{2}\right) u_{i . j+1}=2\left(1-\frac{\Delta t^{2}}{\Delta x^{2}} \frac{T_{0}}{\rho A}\right) u_{i, j}+\frac{\Delta t^{2}}{\Delta x^{2}} \frac{T_{0}}{\rho A} u_{i+1, j}+\frac{\Delta t^{2}}{\Delta x^{2}} \frac{T_{0}}{\rho A} u_{i-1, j}+\left(-1+\frac{C}{\rho A} \frac{\Delta t}{2}\right) u_{i . j-1}
$$

where $\Delta t$ is the time step, $\Delta x$ is the element string length, $u_{i, j}$ is the string displacement, and the subscripts $i$ and $j$ represent the number of a lattice point in the space and time coordinates, respectively.

The response at each time step is calculated using Eq. (22). The string displacement should be monitored, while the contact with and separation from the vibration suppressor are determined at each time step.

\section{Results and Discussion}

\subsection{Load acting on the vibration suppressor}

An example of the calculated results of the load acting on the vibration suppressor using Eqs. (9) and (17) is shown in Fig. 9. This figure shows that the load acting on the vibration suppressor is zero at $t / T_{n 0}=0.5$. Moreover, when there is a gap, the duration for which the load is applied becomes short.
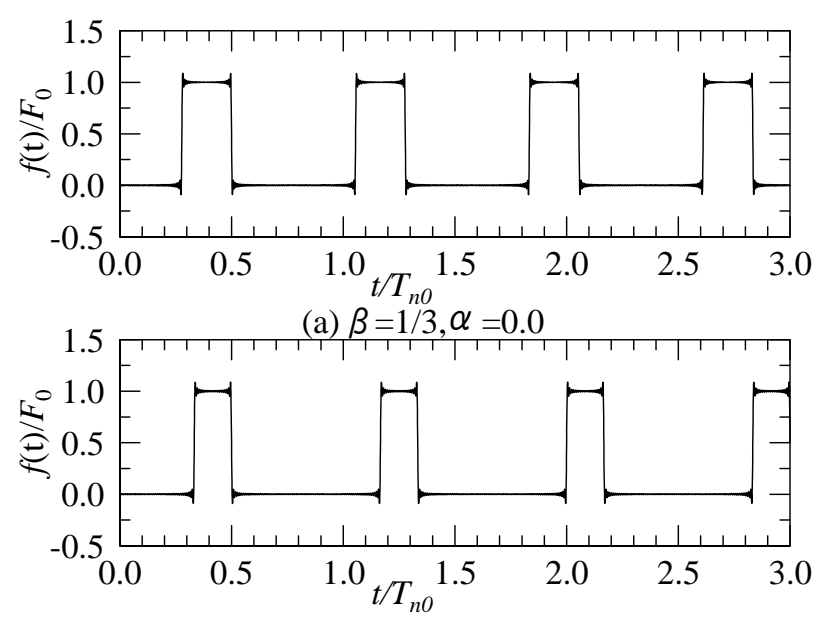

(b) $\beta=1 / 3, \alpha=0.25$

Fig. 9 Time history of reaction force

\subsection{String displacement distribution and time history of the string displacement}

Figures 10-15 show the displacement distributions during one natural period and the time history of the string displacement after releasing the string. In Figs. 11, 13, and 15, the calculated results using the exact solution and the finite difference analysis are plotted with solid lines and dashed lines, respectively. Both results are in good agreement. Figures 10, 12, and 14 show that the inclination of the string after collision is equal to the initial string inclination. The

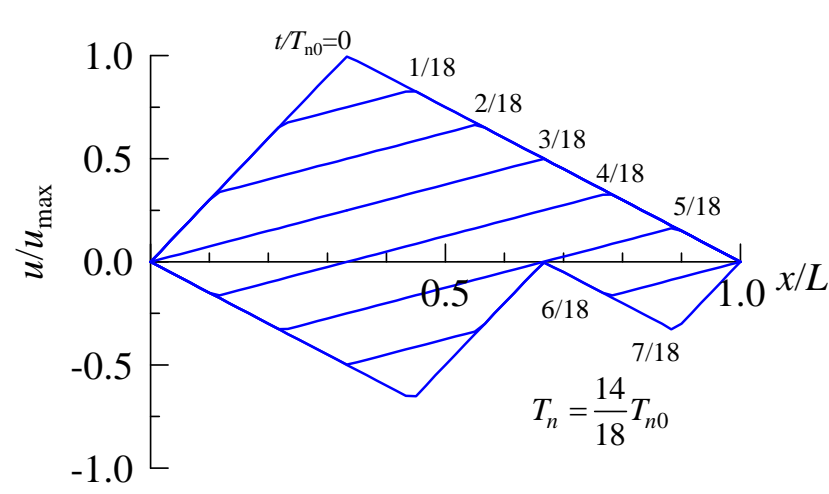

Fig. 10 Vibration mode of string with vibration suppressor $(\beta=1 / 3, \alpha=0.0)$

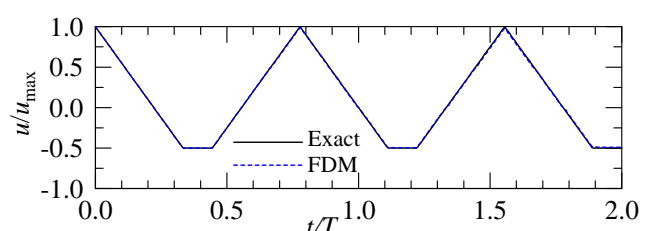

(a) $x / L=1 / 3$

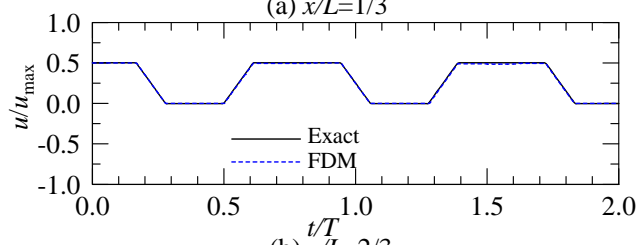

(b) $x / L=2 / 3$

Fig. 11 Time history of string displacement

$$
(\beta=1 / 3, \alpha=0.0)
$$




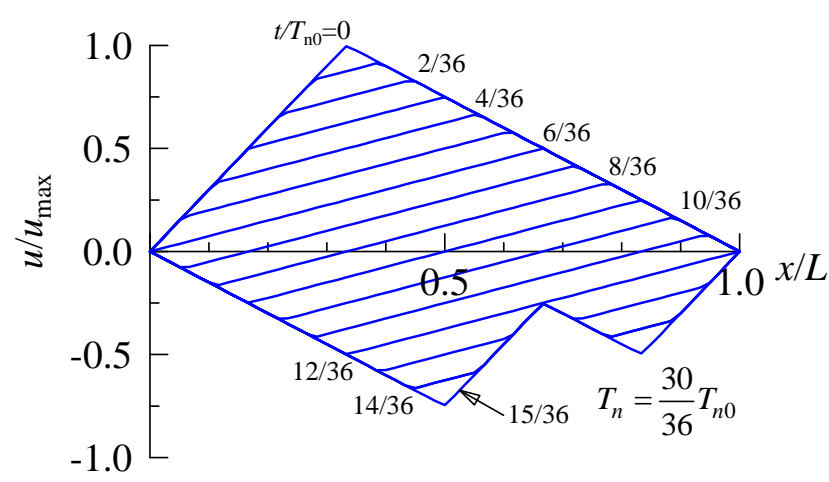

Fig. 12 Vibration mode of string with vibration suppressor ( $\beta=1 / 3, \alpha=0.25$ )

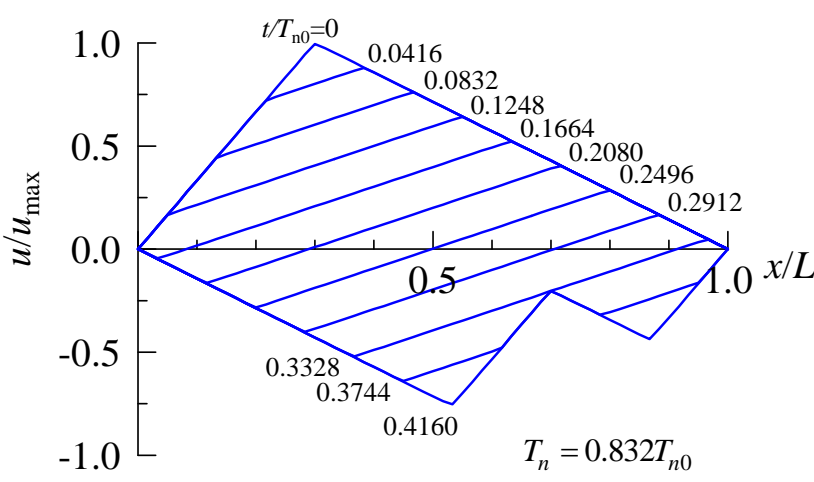

Fig. 14 Vibration mode of string with vibration suppressor ( $\beta=0.3, \alpha=0.2$ )

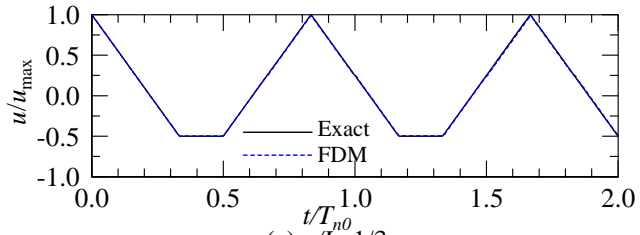

(a) $x / L=1 / 3$

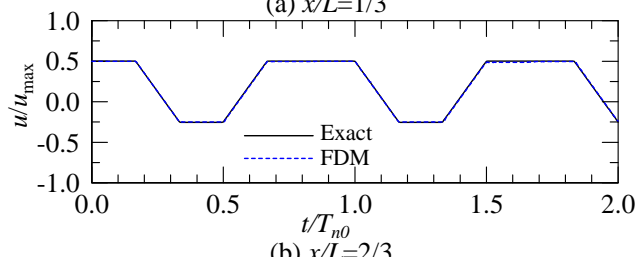

(b) $x / L=2 / 3$

Fig. 13 Time history of string displacement $(\beta=1 / 3, \alpha=0.25)$

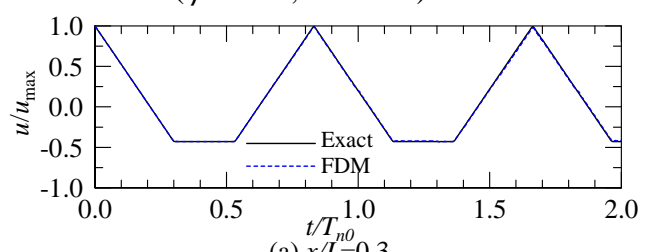

(a) $x / L=0.3$

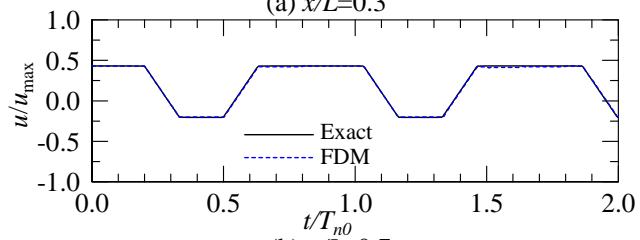

(b) $x / L=0.7$

Fig. 15 Time history of string displacement $(\beta=0.3, \alpha=0.2)$

number of the terms, $i$ and $k$, is up to 256 in the calculation of the exact solution in Eqs. (11) and (19). The divided number of the string is 600 and the time step $\Delta t / T_{n 0}$ is 0.004 in the calculation of the finite difference analysis. The string displacement at the vibration suppressor is monitored at each time step. If $u((1-\beta) L, t)$ is less than $\delta_{0}$, $u((1-\beta) L, t)=\delta_{0}$ is used in the subsequent calculation. As shown in these figures, the natural period of the string with the vibration suppressor is $\{1-\beta(1-\beta)(1-\alpha)\} T_{n 0}$, where $T_{n 0}$ is the natural period of the string without the vibration suppressor.

\subsection{Natural frequency}

When the position of the vibration suppressor is opposite to the pulled position, the natural frequency of the string depends on the pulled position and the gap between the string and the vibration suppressor. The calculated results regarding the effects of parameters $\beta$ and $\alpha$ on the natural frequency of the string with the vibration suppressor are

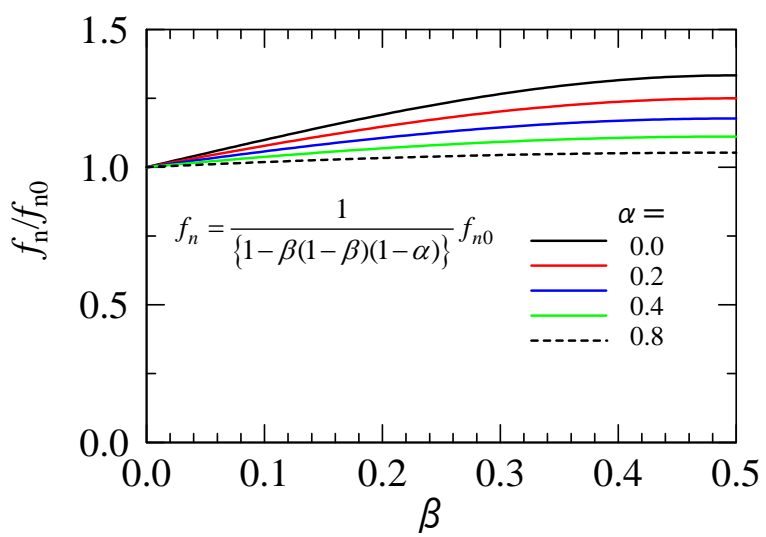

Fig. 16 Natural frequency of string with vibration suppressor 
shown in Fig. 16. This figure shows that the use of a vibration suppressor leads to an increase in the natural frequency of the string. The frequency ratio becomes maximum value $f_{n} / f_{n 0}=4 / 3=1.333$ at $\beta=0.5$ and $\alpha=0.0$.

\section{Conclusions}

In this paper, an exact solution for the free vibration of a string when the position of the vibration suppressor is opposite to the pulled position is presented. A finite difference analysis of the string vibration is also performed considering the vibration suppressor. The following results were obtained:

(1) The exact solution for the free vibration of the string with a vibration suppressor is obtained using the Fourier series expansion of the load acting on the vibration suppressor.

(2) The exact solution is confirmed by the calculation of the finite difference analysis.

(3) The use of a vibration suppressor leads to an increase in the natural frequency of the string. Moreover, the natural frequency depends on the pulled position and the gap between the string and the vibration suppressor.

\section{Acknowledgment}

This work was supported by JSPS KAKENHI Grant Number15K05859.

\section{References}

Katsuno, Y., Matsuhisa, H., Utsuno, H., Yamada, K. and Sawada, K., Non-contact vibration control of elevator rope using magnetic force -Comparison between modal analysis and transfer matrix analysis-, Proceeding of the Dynamics and Design Conference 2007, No. 07-8(2007),CD-ROM (Paper No. 426) ,pp.1-6 (in Japanese).

Kimura, H., Nakagaki, S., Osada, A., Munakata, T. and Nagata, K., Vibration Analysis and Suppression of Elevator Rope for High-Rise Building, Proceedings of the 3rd World Conference on Structural Control, Vol. 3 (2002), pp.827-832.

Kimura, H. and Nakagawa, T., Vibration Analysis of Elevator Rope with Vibration Suppressor, Journal of Environment and Engineering, Vol. 2, No. 1 (2007a), pp. 76-86. DOI: 10.1299/jee.2.76.

Kimura, H., Ito, H., Fujita, Y. and Nakagawa, T., Forced Vibration Analysis of an Elevator Rope with Both Ends Moving, Transactions of ASME, Journal of Vibration and Acoustics, Vol. 129, No. 4 (2007b), pp.471-477. DOI:10.1115/1.2748471.

Kimura, H., Ito, H. and Nakagawa, T., Vibration Analysis of Elevator Rope (2nd Report, Forced Vibration of Rope with Damping), Journal of System Design and Dynamics, Vol. 2, No. 2 (2008), pp.540-549. DOI: 10.1299/jsdd.2.540.

Kimura, H., Free Vibration of Elevator Rope with Vibration Suppressors (When Vibration Suppressors are arranged at Equal Intervals), Transactions of the Japan Society of Mechanical Engineers, Series C, Vol. 79, No. 802 (2013), pp. 1904-1912 (in Japanese).

Kimura, H., Free Vibration Analysis of a String with Vibration Suppressor, Transactions of the JSME (in Japanese), Vol. 80, No. 812 (2014a). DOI:10.1299/transjsme.2014trans0059.

Kimura, H., Free Vibration Analysis of an Elevator Rope with Vibration Suppressor Located at Center of the Rope, Proceedings of the 23th Transportation and Logistics Conference 2014, Vol. 14, No. 65 (2014b), pp. 95-98 (in Japanese).

Nakagawa, J. and Arai., S., Damping of elevator rope swing by resting movement of compensation sheave, Elevator, Escalator and Amusement Rides Conference, No. 11-94 (2012), pp. 23-26 (in Japanese).

Nakagawa, T. Nishikawa, T. and Kimura, H., High-Reliability Technologies for World's Fastest Elevators, Toshiba Review, Vol. 60, No. 5 (2005), pp. 26-29 (in Japanese).

Otsuki, M., Yoshida, K., Nagata, K., Kimura, H. and Nakagawa, T., Vibration control for rope-sway of elevator of high-rise building (Application of nonstationary optimal control to time-varying flexible system), Transactions of the Japan Society of Mechanical Engineers, Series C, Vol. 68, No. 676 (2002), pp. 3589-3596 (in Japanese).

Watanabe, S., Nishida, T. and Yamane, T., Elevator rope dynamics during long-period ground motion, Elevator, Escalator and Amusement Rides Conference, No. 06-67 (2007), pp. 15-18 (in Japanese).

Yasuda, K., Vibration Engineering (Fundamentals) (2000), pp. 143-144, Corona Publishing Co., LTD (in Japanese). 